\title{
Case Report: The Paratesticular Rhabdomyosarcoma in Children
}

\author{
Manal Azizi*, Abdeladim Babakhouya, Maria Rkain, Noufissa Benajiba \\ Hematology and Oncology Unit, Department of Pediatric, UHC Mohammed VI, University Mohammed I, Oujda, Morocco \\ Email: *ma_az20@hotmail.fr
}

How to cite this paper: Azizi, M., Babakhouya, A., Rkain, M. and Benajiba, N. (2021) Case Report: The Paratesticular Rhabdomyosarcoma in Children. Journal of Cancer Therapy, 12, 358-364. https://doi.org/10.4236/jct.2021.126032

Received: April 27, 2021

Accepted: June 8, 2021

Published: June 11, 2021

Copyright (c) 2021 by author(s) and Scientific Research Publishing Inc. This work is licensed under the Creative Commons Attribution International License (CC BY 4.0).

http://creativecommons.org/licenses/by/4.0/ (c) (i) Open Access

\begin{abstract}
Rhabdomyosarcoma is a rare and aggressive mesenchymal tumor, whose paratesticular location is the most common genitourinary site. It is the prerogative of the child and the young adult. On physical examination, a painless scrotal tumefaction is observed. His diagnosis is essentially provided by testicular ultrasound. His therapeutic strategy is codified and based on the combination of chemotherapy and surgical excision or radiotherapy. The precocity of the management and the accuracy of the extension and radiology tests are important for the vital and functional prognosis. We report a case of paratesticular rhabdomysarcoma of alveolar type, diagnosed in front of a large left painless scrotal mass, which has been evolving for less than one month in a 7-year-old child. An inguinal orchiectomy was performed. A histological examination of the excised tissue revealed an epididymal RMS of the alveolar type. Our patient had 9 chemotherapy sessions with vincristine, actinomycin $\mathrm{C}$ and cyclophosphamide. Each chemotherapy session took place over four days, with a 21-day cycle. He was evaluated for 4 years and demonstrated good clinical improvement. The experience gained from the index case is crucial for the management of patients with intratesticular rhabdomyosarcoma who must always ensure that the radical inguinal orchiectomy with lymph node dissection is covered by chemotherapy and/or radiotherapy. This will potentially reduce the chances of tumor recurrence and/or metastasis, thus improving patient prognosis.
\end{abstract}

\section{Keywords}

Rhabdomyosarcoma, Tumor Paratesticular, Treatment

\section{Introduction}

Paratesticular Rhabdomyosarcoma (RMS) is very rare malignant tumors arising 
from the mesenchymal tissues of tunica, epididymis or spermatic cord. They are aggressive tumors especially in children. It requires early diagnosis, accurate clinical and radiological extension assessment and well-codified treatment based on the pathological stage [1]. Paratesticular rhabdomyosarcomas typically present as a unilateral, painless scrotal swelling or mass. Lymph node metastasis develops in approximately one third of cases with paratesticular lesions [2]. Radical orchidectomy procedure with negative surgical margins is gold standard in the treatment of these tumors. Chemotherapy is recommended for the control of retroperitoneal micrometastases.

We report a case of paratesticular rhabdomyosarcoma in children, of fortuitous discovery, with the aim of sensitizing practitioners to this pathology that must be evoked in front of a voluminous painless scrotal bursa.

\section{Case Report}

This is a 7-year-old child, admitted for the management of a large, non-painful left bursa that has been in progress for less than a month. He had no history of trauma, difficulty in passing urine or blood in urine. The parents denied any history of cancer in the family. His past medical, social, family and environmental history were uneventful. They reported that, the patient received Diphtheria, Tetanus, Pertussis and Haemophilus influenza and Hepatitis B, pneumo-conj, and Measle-Rubella (MR), tuberculosis vaccines at the right schedules.

Clinical examination finds an apyretic patient in good general condition, His weight and height were $20.2 \mathrm{~kg}$ and $86 \mathrm{~cm}$ respectively, with a pulse and blood pressure of 68 beats per minute and 127/73 mmHg respectively. The left testicle was swollen, painless and non-tender, lumpy and of a firm consistency, of regular contours, and without local inflammatory signs (Table 1). The right testicle is without abnormality. His neurological examination revealed normal motor function (normal gait and muscle tone), normal sensory function, and normal

Table 1. Socio-demographic and clinical characteristics.

\begin{tabular}{cc} 
Sex & Male \\
Age & 7 years \\
Residence & Morocco \\
Urban/rural & Urban \\
Vaccine & Yes \\
Consanguinity & No \\
Weight & $20.2 \mathrm{~kg}$ \\
Height & $86 \mathrm{~cm}$ \\
Blood pressure & $127 / 73$ mmHg \\
Antecedents & Without \\
Dianostic delay & One month \\
Clinical signs & Swollen left testicle, painless and non-tender, lumpy, \\
& of a firm consistency, and of regular contours. \\
\hline 359 & \\
\hline
\end{tabular}


reflex responses. The bilateral inguinal lymph nodes were unpalpable. No significant findings were detected on the other physical examinations.

Testicular CT revealed a left epididymal tumor mass pushing back the ipsilateral testis, measuring $14-21 \mathrm{~mm}$, with no signs of locoregional invasion or deep lymphadenopathy. There is no vaginal effusion. The testis and the right epididymis are normal.

Tumor markers (beta-HCG and alpha-foetoprotein) are normal; free chorionic gonadotropic hormone (beta-HCG) $<0.04 \mathrm{mUI} / \mathrm{ml}$, serum alpha-fetoprotein $(\mathrm{AFP})=1.46 \mathrm{ng} / \mathrm{ml})$.

An inguinal orchidectomy is performed after first and high ligature of the spermatic cord with full resection margin (IRS Group 1). Histological and immunohistochemical examination of the orchiectomy piece shows an epididymal RMS of the alveolar type.

For the extension assessment, abdominal ultrasound as well as thoraco-abdominal-pelvic computed tomography are normal, including no retroperitoneal or mediastinal lymphadenopathy or liver metastases. The chest X-ray, myelogram and biological assessment (hemograms, renal and hepatic function) were normal.

The patient was classified high risk group $\mathrm{D}$ due to the adverse histological type according to the protocol RMS 05 despite N0, complete margins, small tumor, and age less than 10 years.

The patient was treated with adjuvant polychemiotherapy implemented in 9 cures according to the MMT 2005 protocol, combining Ifosfamide $\left(3 \mathrm{~g} / \mathrm{m}^{2}\right)$, Vincristine $\left(1.5 \mathrm{mg} / \mathrm{m}^{2}\right)$ and Actinomycin $\mathrm{D}\left(1.5 \mathrm{mg} / \mathrm{m}^{2}\right)$. Each treatment is spread over 4 days and the cycles are spaced by 21 days.

The evolution was marked by a convulsive condition that required an intensive care stay for 5 days with intubation; the biological assessment showed a corrected hyponatremia at $115 \mathrm{mmol} / \mathrm{l}$.

The brain CT scans was without abnormality. Subsequently, the patient developed acute paraparesis. A medullary MRI was in favor of lumbar epiduritis (Figure 1 and Figure 2) treated with corticosteroid bolus for 3 days with oral relay for 3 months; furthermore the cytospin did not find any tumor cells.

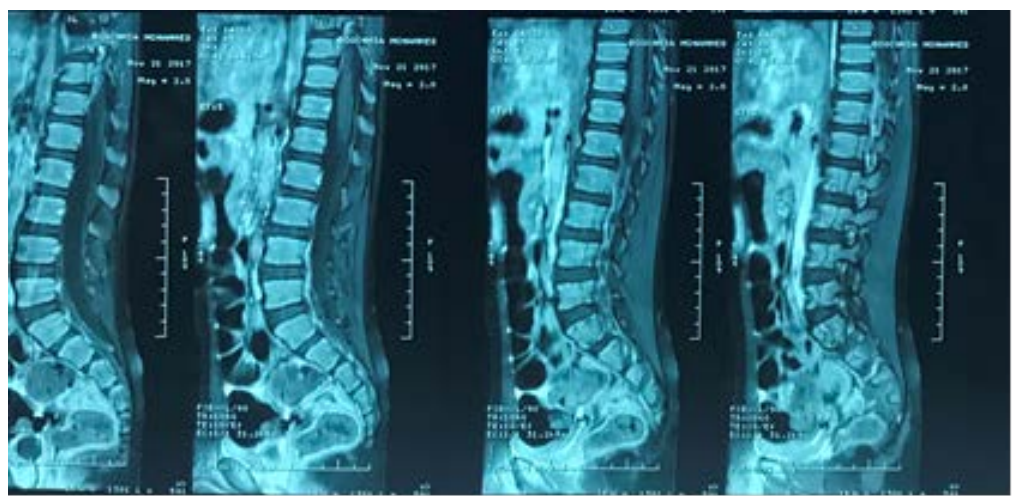

Figure 1. MRI Medullary, sequence T1 injection. Extended lumbar epidural contrast to foramen of bilateral conjugation and around the roots of ponytail. 


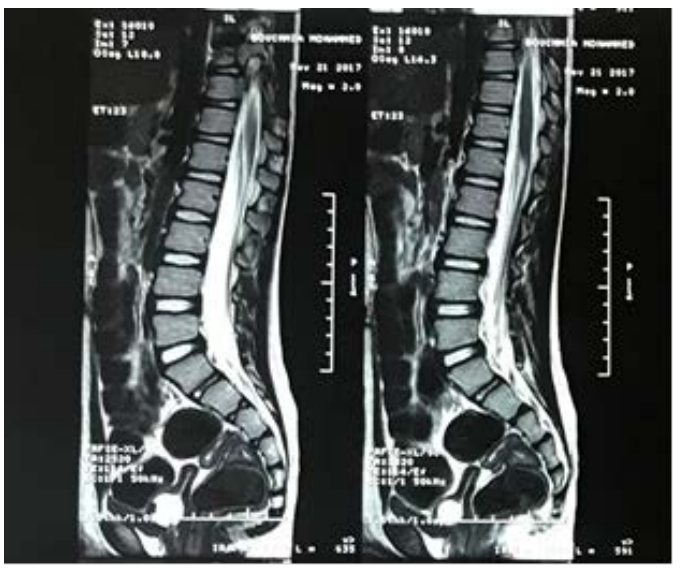

Figure 2. MRI medullary, sequence T2. Epidural lumbar contrast, extended to foramens of conjugation in bilateral and around the roots of ponytail. Absence of signal abnormality or intramedullary pathological contrast.

After a multidisciplinary consultation meeting, radiotherapy had no indication for our patient because of the paratesticular site, the complete resection (no residual foci) and the absence of a retroperitoneal ganglion.

The MRI medullary control revealed regression of lumbar epiduritis after treatment.

The patient is currently following a monitoring protocol established as follows: monthly during the first year, bimonthly during the following year, and annually for a period of 5 years. The trend is favorable after 4 years of decline.

\section{Discussion}

Paratesticular RMS develops from the mesenchymal tissues of the spermatic cord, epididymis and testicular tunics [2]. It represents 7\% of rhabdomyosarcomas all locations combined [3].

Paratesticular RMS is observed at any age, but especially in children and young adults. There are two peaks of incidence; one at the age of 4 and the other at the age of 16 [4].

Clinically paratesticular rhabdomyosarcomas have the same symptomatology as other tumors with intrascrotal development. It is often a large, painless bursa, most often discovered accidentally, sometimes with simple scrotal pain [5]. The testicular examination should be bilateral and attentive. It eliminates the main causes of painless large bursa including hydrocele, cord cyst, epididymitis or inguino-scrotal hernia which could lead to detrimental delay in diagnosis. It shows a hard painless mass of variable topography. The paratesticular site of the tumor is sometimes difficult to determine by examining the scrotum. This clinical examination will be completed by examination of the lymph node areas and a general examination for metastases.

Any suspicion of a tumor requires the prescription of a bilateral scrotal ultrasound [5] [6], objectiving a heterogeneous echostructural mass with inguinoscrotal extension in $80 \%$ of cases. The dosage of tumor markers (beta-HCG, LDH 
and alfa-foeto-protein) should be part of the systematic assessment of any intrascotal tumor, but it is often normal in paratesticular tumors [2].

The diagnostic confirmation is then based on the pathological examination of the orchidectomy piece performed inguinal [7], showing rhabdomyoblasts characteristic of rhabdomyosarcoma.

Thoraco-abdominal-pelvic CT with injection of contrast product is recommended for tumor extension assessment; (evaluation of pelvic and lumbo-aortic glands and hepatic or pulmonary metastases) [5] [8].

Paratesticular rhabdomyosarcoma is very lymphophilic, especially for the lumbo-aortic chain lymph nodes. Some authors report that lymph node invasion occurs in $40 \%$ of cases, pulmonary metastases occur in 54\% [9], lumbar cord involvement is observed in $27 \%$ to $40 \%$ of cases [5].

In our observation, the chest X-ray was normal. Other tests may be prescribed if there is any doubt about the diagnosis. Magnetic resonance imaging (MRI) can better appreciate the relationship between the tumor and the epididymis and locate it within components of the scrotum [10].

There are classically three histological subtypes of RMS:

- The embryonic type which represents $97 \%$ of cases, the most frequent variety, preferentially affects children under 10 years old and presents the best prognosis [7] [9].

- The alveolar type which represents $20 \%$ of histologies in children, but this rate increases in the series of rhabdomyosarcoma in adults. It is considered to have a poor prognosis [11].

- The pleomorphic type, which is a form of adults over 45 years old, the frequency of which increases with age [12].

Treatment is based on surgery (inguinal orchiectomy with upper and first ligation of the spermatic cord [7], polychemotherapy and radiotherapy. Polychemotherapy contributes to improving survival and reducing the frequency of relapses [13]. It also makes it possible to reduce the indications for very extensive surgical excision. Protocol times range from 18 to 24 months. These protocols are: the VAC, IVA and VIE protocol with (V: vincristine, A: actinomycin D, E: etoposide, I: ifosfamide and C: cyclophosphamide). The combination (vincristine ( 1 to $2 \mathrm{mg} / \mathrm{m}^{2}$ ), actinomycin D (1500 to $2500 \mathrm{mg} / \mathrm{m}^{2}$ ) and cyclophosphamide $\left(1\right.$ to $\left.2 \mathrm{mg} / \mathrm{m}^{2}\right)$ ) is the most widely used and is based on the administration of several treatments spread over 5 days and spaced 2 to 4 weeks apart. In the event of a tumor resistant to this multidrug therapy, cisplatin, bleomycin and doxorubicin are used [5].

In certain cases, radiotherapy is carried out in addition to chemotherapy and curage on the residual tumor sites, on the retro-peritoneal nodes and on metastases, in particular pulmonary ones. The doses used depend on the pathological stage and are spread over several weeks [14].

The therapeutic indications depend on the pathological stage according to the IRS classification (Intergroup Rhabdomyosarcoma Study) [15]:

- Group I: orchiectomy followed by chemotherapy, no radiotherapy. 
- Groups II and III: This treatment is supplemented by retroperitoneal lymphonodal therapy combined with local radiotherapy.

- Group IV: orchidectomy followed by chemotherapy without lymphonodal curage with removal of metastasis if technically feasible, supplemented by radiotherapy directed at retroperitoneal lymphonodes and metastases.

The prognosis of paratesticular RMS depends on the patient's age, histological type, pathological stage and response to treatment. The survival rate exceeds $90 \%$ at 5 years for stages I and II [14].

\section{Conclusion}

Paratesticular rhabdomyosarcoma is a rare tumour that requires early diagnosis and accurate extension assessment to promote curative treatment. After orchiectomy, the therapeutic strategy is currently well codified, combining lymph node curage, polychimiotherapy and radiotherapy according to tumor stage and prognostic group. Strict follow-up has to be instituted for all patients.

\section{Conflicts of Interest}

The authors declare that they have no conflict of interest.

\section{References}

[1] Radouane, B., El Fenni, J., Chaouir, S., Amil, T., Hanine, A. and Ben Ameur, M. (2004) Paratesticular Rhabdomyosarcoma. A Case Report. Journal de Radiologie, 85, 779-781. https://doi.org/10.1016/S0221-0363(04)97683-4

[2] Bennani, H., El Ghanmi, J., Karmouni, T., El Khader, K., Koutani, A. and Iben Attya Andaloussi, A. (2014) Rhabdomyosarcome paratesticulaire (RMSP) multimétastatique: À propos d'un cas. Canadian Urological Association Journal, 8, 660-662. https://doi.org/10.5489/cuaj.219

[3] Silvestris, N., Zito, F.A., Resta, L., Popescu, O., Popescu, O., Rossi, R., et al. (2009) Unique Case of Giant Adult Paratesticular Spindle Cell Rhabdomyosarcoma. Urology, 73, 500-502. https://doi.org/10.1016/j.urology.2008.09.025

[4] Wu, H.Y., Snyder, H.M. and Womer, R.B. (2009) Genitourinary Rhabdomyosarcoma: Which Treatment, How Much, and When? Journal of Pediatric Urology, 5, 501 506. https://doi.org/10.1016/j.jpurol.2009.06.011

[5] Faure, A., Diakité, M.L., Panait, N., Chaumoître, K., Rome, A. and Merrot, T. (2012) Le rhabdoyosarcome para-testiculaire de l'enfant: Une urgence scrotale. Archives de Pédiatrie, 19, 1340-1344. https://doi.org/10.1016/j.arcped.2012.09.022

[6] Mak, C.W., Chou, C.K., Su, C.C., Huan, S.K. and Chang, J.M. (2004) Ultrasound Diagnosis of Paratesticular Rhabdomyosarcoma. The British Journal of Radiology, 77, 250-252. https://doi.org/10.1259/bjr/20564274

[7] Dasgupta, R. and Rodeberg, D.A. (2012) Update on Rhabdomyosarcoma. Seminars in Pediatric Surgery, 21, 68-78. https://doi.org/10.1053/j.sempedsurg.2011.10.007

[8] Zheng, L., Tang, H., Chen, X., Yang, H. and Yang, M. (2013) Paratesticular FetalType Rhabdomyoma in a 12-Year-Old Boy: A Case Report and Literature Review. Urology, 82, 1150-1152. https://doi.org/10.1016/j.urology.2013.04.029

[9] Tazi, K., Moudouni, S., Koutani, A., Ibn Attya, A., Hachimi, M. and Lakrissa, A. (2000) Rhabdomyosarcome Paratesticulaire de l'adulte jeune. Progrès en Urologie, 
$10,469-472$.

[10] Mason, B.J. and Kier, R. (1998) Sonographic and MR Imaging Appearances of Paratesticular Rhabdomyosarcoma. American Journal of Roentgenology, 171, 523-524. https://doi.org/10.2214/ajr.171.2.9694492

[11] Huh, W.W. and Skapek, S.X. (2010) Childhood Rhabdomyosarcoma: New Insight on Biology and Treatment. Current Oncology Reports, 12, 402-410. https://doi.org/10.1007/s11912-010-0130-3

[12] Routh, J.C., Dasgupta, R., Chi, Y.Y., Shnorhavorian, M., Tian, J, Walterhouse, D.O. et al. (2020) Impact of Local Control and Surgical Lymph Node Evaluation in Localized Paratesticular Rhabdomyosarcoma: A Report from the Children's Oncology Group Soft Tissue Sarcoma Committee. International Journal of Cancer, 147, 3168 3176. https://doi.org/10.1002/ijc.33143

[13] Rodeberg, D. and Paidas, C. (2006) Childhood Rhabdomyosarcoma. Seminars in Pediatric Surgery, 15, 57-66. https://doi.org/10.1053/j.sempedsurg.2005.11.009

[14] Ghorbal, L., Abid, W., Elloumi. F., Sallemi, T., Frikha, M. and Daoud, J. (2015) Rhabdomyosarcome embryonnaire paratesticulaire: À propos d'un cas et revue de la littérature. Cancerl Radiothérapie, 19, 334-336.

https://doi.org/10.1016/j.canrad.2015.05.002

[15] Beverly Raney, R., Maurer, H.M., Anderson, J.R., Andrassy, R.J., Donaldson, S.S., Qualman, S.J., et al. (2001) The Intergroup Rhabdomyosarcoma Study Group (IRSG): Major Lessons from the IRS-I through IRS-IV Studies as Background for the Current IRS-V Treatment Protocols. Sarcoma, 5, Article ID: 925281.

https://doi.org/10.1080/13577140120048890 